8-1-2019

\title{
Conocimiento, actitudes y prácticas sobre el Zika: Implicancias programaticas para la prevencion del Zika en Republica Dominicana
}

Breakthrough RESEARCH

Follow this and additional works at: https://knowledgecommons.popcouncil.org/departments_sbsr-pgy How does access to this work benefit you? Let us know!

\section{Recommended Citation}

Breakthrough RESEARCH. 2019. “Conocimiento, actitudes y prácticas sobre el Zika: Implicancias programaticas para la prevencion del Zika en Republica Dominicana," Programmatic Research Brief. Washington, DC: Population Council. 


\section{Antecedentes}

El primer brote de Zika registrado en las Américas ocurrió en 2015, cuando hubo un alza en malformaciones congénitas y otras complicaciones neurológicas. ${ }^{1}$ En abril del 2016, el Departamento de Estado de los Estados Unidos destinó fondos para una Respuesta al Zika de la USAID, priorizando, en países afectados, esfuerzos programáticos orientados a minimizar los efectos negativos del Zika durante el embarazo.

Un elemento central de la Respuesta al Zika de la USAID son los programas de cambios sociales y de comportamiento, que tienen como objetivo promover prácticas y comportamientos efectivos que reduzcan la transmisión del Zika. Estos programas enfatizan la protección personal para mujeres embarazadas, involucran a comunidades de alto riesgo en prácticas de prevención, y mejoran el acceso de mujeres a servicios de atención prenatal.*

\section{¿Por qué recopilamos información sobre conocimiento, actitudes y prácticas?}

Las teorías de cambio de comportamiento nos ayudan a entender qué factores facilitan y motivan a las personas a comportarse de una manera u otra. ${ }^{4} \mathrm{El}$ modelo socioecológico de cambio de comportamiento destaca la importancia de factores individuales (como el conocimiento, las percepciones y las actitudes sobre una enfermedad y su prevención), factores comunitarios (como el ambiente normativo) y factores sociales y estructurales (como el acceso a recursos y servicios) para entender si una persona realiza o no un comportamiento en particular. Otro modelo-Extended Parallel Processing Model-destaca que la percepción de riesgo que una persona asocia con una enfermedad, como también su confianza en la efectividad de una posible solución y su compromiso con practicarlas, impactará en que una persona realice prácticas preventivas o no. ${ }^{4}$ Esta y otras teorías ofrecen una base para medir indicadores que permiten que ejecutores de programas de cambio social y de comportamiento decidan las mejores estrategias para impactar el comportamiento de comunidades específicas.

\section{¿Cómo se recopilaron los datos?}

En el año 2018, el proyecto Breakthrough RESEARCH realizó una encuesta transversal de hogares en Guatemala, Honduras, El Salvador y la República Dominicana. La encuesta evaluó, entre otros factores, el conocimiento, las actitudes y los comportamientos (CAP) relacionados al Zika y a otras enfermedades transmitidas por el mosquito Aedes aegypti. Se utilizó un diseño muestral aleatorio, multietápico y por conglomerado. Se encuestaron hombres y mujeres de entre 18

\footnotetext{
* Para mayor información visite: https://www.usaid.gov/what-we-do/ global-health/zika.
}

\section{¿POR QUÉ IMPORTA EL ZIKA?}

- El virus del Zika es una enfermedad contagiosa transmitida por los mosquitos Aedes aegypti, que también transmiten otros arbovirus, como el dengue y la chikunguña.'

- El Zika también se puede transmitir a través de relaciones sexuales y de una madre embarazada a su feto. ${ }^{1}$ Se sabe que el Zika puede causar problemas neurológicos, como el síndrome congénito por el virus del Zika. ${ }^{2,3}$

- Muchas personas infectadas con el virus del Zika no desarrollan síntomas, lo que genera una percepción de riesgo menor. ${ }^{2}$

y 49 años, residentes de áreas de intervención de programas de la USAID. En la República Dominicana, la encuesta fue realizada en agosto del 2018 en las áreas de intervención del programa de Respuesta al Zika de la USAID: Puerto Plata, Santiago, La Vega, Azua, San Cristóbal, La Altagracia, La Romana, Santo Domingo, y Distrito Nacional. Se entrevistaron 651 personas, de las cuáles el 34 por ciento eran hombres y el 66 por ciento eran mujeres. Los datos se ponderaron según características sociodemográficas a modo de reflejar la población de las áreas de intervención.

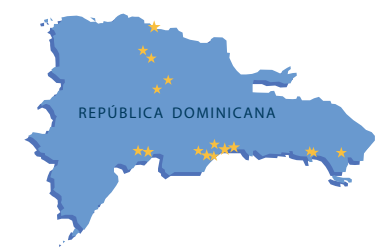

El estudio recogió información sobre cuatro grandes dimensiones:

- Información sociodemográfica: Edad, educación, sexo, bienes del hogar.

- Conocimiento: Transmisión, efectos en la salud, prevención de cada enfermedad.

- Actitudes: Percepciones de riesgo de la enfermedad, efectividad de comportamientos preventivos, factibilidad de llevar a cabo conductas preventivas.

- Autorreporte de conductas preventivas: Comportamientos para prevenir picaduras de mosquitos y criaderos de mosquitos en los últimos siete días y en los últimos treinta días.

- Prácticas observadas: Identificación del uso de tapas seguras para el almacenamiento de agua donde suelen estar los criaderos de mosquitos. 


\section{Implicancias programáticas claves}

Estas recomendaciones para programas que impulsen cambios sociales y de comportamiento están basadas en hallazgos de las encuestas realizadas en la República Dominicana.

1. Los programas y mensajes de prevención debiesen destacar los vínculos entre el virus del Zika y posibles defectos congénitos, incluyendo el síndrome congénito por el virus del Zika, especialmente entre la población masculina. Esto distingue al Zika de otros arbovirus. Para mujeres embarazadas y sus parejas, tener un hijo saludable puede ser una motivación fundamental para prevenir el virus.

2. Los programas de prevención debiesen priorizar comportamientos que cuentan con evidencia de su efectividad contra las enfermedades transmitidas por el Aedes-aegypti; y los mensajes debiesen destacar esta evidencia e incluir instrucciones detalladas para comportamientos preventivos complejos.

3. Los mensajes de prevención debiesen continuar destacando el rol de la transmisión sexual del Zika, especialmente durante el embarazo.

4. Los programas de prevención debiesen destacar y diseñar maneras de simplificar los comportamientos deseados, de manera que sean más comprensibles y factibles.

5. Los programas de prevención debiesen aprovechar el conocimiento que existe sobre la transmisión del dengue mediante mosquitos, para informar sobre enfermedades menos conocidas que también son transmitidas por el mosquito Aedes-aegypti. Relacionar las distintas enfermedades puede ayudar a fortalecer el conocimiento de la población respecto al modo de contagio del Zika y del chikunguña. Sin embargo, se debe además destacar el riesgo adicional del Zika durante el embarazo.

6. Los mensajes de prevención debiesen especificar que los mosquitos pueden picar a cualquier hora, a modo de destacar que las estrategias de protección personal, como repelentes, deben usarse durante el transcurso de todo el día.

7. Debiesen existir programas de prevención dirigidos específicamente a hombres, ya que reportan peores niveles de conocimientos y actitudes sobre el Zika. Esto es de suma importancia, dado el papel que tienen los hombres en el uso de condones como mecanismo de prevención.

8. Los mensajes de prevención mediante visitas presenciales de educadores en los hogares son una estrategia que sirve para aumentar el conocimiento de las personas y promover conductas preventivas.

\section{Hallazgos clave sobre el conocimiento del Zika}

\section{Conocimiento de la enfermedad}

La mayoría de las personas encuestadas habían escuchado del Zika, dengue o chikunguña, y al menos tres de cada cuatro personas habían escuchado sobre las tres enfermedades (ver Tabla 1). Sin embargo, de las tres, el Zika era la enfermedad menos conocida, especialmente entre hombres. Aproximadamente un 78 por ciento de los hombres había escuchado del Zika, mientras que 90 por ciento de las mujeres había escuchado del Zika, una cantidad significativamente mayor $(p<0.01){ }^{+}$

TABLA 1. CONOCIMIENTO DE ENFERMEDADES TRANSMITIDAS POR EL MOSQUITO AEDES AEGYPTI

\begin{tabular}{l|c} 
ENFERMEDAD & $\begin{array}{c}\text { \% DE PERSONAS QUE HAN } \\
\text { ESCUCHADO DE LA ENFERMEDAD }\end{array}$ \\
\hline Zika & $85 \%$ \\
\hline Dengue & $95 \%$ \\
\hline Chikunguña & $94 \%$
\end{tabular}

Mecanismos de transmisión del Zika y sus efectos en la salud

Mediante mosquitos: Entre las personas que conocían las enfermedades, casi todas sabían que los mosquitos transmiten el dengue (90 por ciento), pero muchos menos sabían que los mosquitos transmiten el Zika (59 por ciento) y el chikunguña (53 por ciento). Un porcentaje significativamente menor de hombres (52 por ciento), en comparación con las mujeres (64 por ciento), sabía que el Zika se transmite mediante una picadura de mosquito $(p<0.05)$. Menos de un tercio $(29$ por ciento) de quienes sabían que el Zika se transmite mediante mosquitos sabían que el mosquito puede picar en cualquier momento durante el día o la noche.

Mediante contacto sexual y transmisión vertical: solo tres por ciento de los encuestados que había escuchado del Zika sabía que se puede transmitir sexualmente y menos de uno por ciento sabía que se puede transmitir de una madre a un feto durante el embarazo.

Solo 10 por ciento de los encuestados que había escuchado del Zika identificó defectos congénitos como un posible efecto adverso del Zika sobre la salud. El nivel educativo de los participantes estaba significativamente relacionado con haber seleccionado defectos congénitos como un posible efecto del Zika: un uno por ciento de las personas con educación primaria sabía de estas consecuencias, mientras un 20 por ciento de

+ Se utilizó la prueba de chi-cuadrado para todos los análisis bivariados. 
las personas con algún nivel de educación universitaria sabía $(p<0.001)$ Este hallazgo probablemente se debe a que individuos con más recursos suelen tener mayor acceso a información.

\section{Métodos de prevención}

Entre los encuestados que conocían el Zika, solo un 61 por ciento conocía al menos un método de prevención de alta efectividad. ${ }^{\ddagger}$

El método de prevención más conocido para reducir el riesgo del Zika fue vaciar agua estancada (44 por ciento), tapar recipientes o contenedores de agua (27 por ciento), usar larvicida en agua almacenada y estancada (27 por ciento), y quitar basura que alberga mosquitos (deschatarrización) afuera del hogar (22 por ciento).

Solo 15 por ciento de los encuestados identificó cepillar o limpiar recipientes de agua, solo 4 por ciento identificó el uso de repelente, y menos de uno por ciento identificó el uso de condones como métodos de prevención del Zika.

En promedio, los hombres estaban significativamente menos informados acerca de los métodos de prevención (en promedio conocían uno) que las mujeres (en promedio conocían dos, $\mathrm{p}<0.001)$.

\section{Hallazgos claves de actitudes sobre el Zika}

\section{Percepciones de riesgo del Zika, dengue y chikunguña}

Como muestra la Figura 1, la mayoría de los participantes no perciben estar en una situación de alto riesgo de contraer Zika,

\section{FIGURA 1. PERCEPCIÓN DE RIESGO POR ENFERMEDAD}

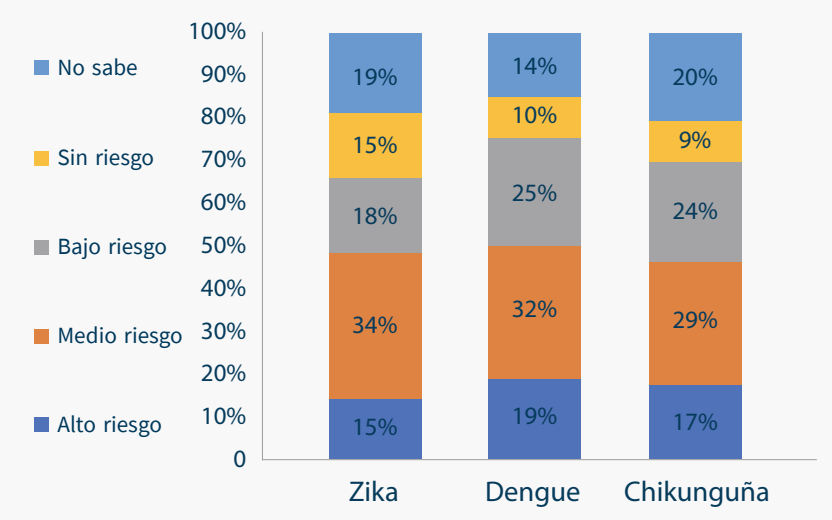

\footnotetext{
‡ Comportamientos efectivos: Usar repelente, vaciar agua estancada, limpiar recipientes y contenedores de agua, tapar recipientes y contenedores de agua, usar larvicida, usar rejillas en ventanas y puertas.
}

dengue, o chikunguña. Más de la mitad de los encuestados (52 por ciento) percibe estar en una situación de bajo riesgo o poco riesgo, o no saben si están o no en una situación de riesgo. Al comparar según nivel de riqueza (medido con un índice de bienes de hogar y servicios), una mayor proporción de los participantes del quintil más bajo o pobre (20 por ciento) no sabían si estaban en una situación de riesgo o no, frente a un 10 por ciento entre el quintil más alto o adinerado (10 por ciento $p<0.05)$.

A pesar de relativamente baja percepción de riesgo de transmisión, la mayoría de los participantes respondió que estarían preocupados si llegasen a contagiarse de Zika (82 por ciento), dengue (89 por ciento), y chikunguña (82 por ciento).

\section{Percepciones de factibilidad de conductas preventivas}

Al responder sobre cómo perciben la factibilidad de las conductas preventivas, ninguna de las alternativas (presentadas en la Tabla 2) fue considerada como muy fácil de llevar a cabo por parte de los encuestados.

\begin{tabular}{|c|c|}
\hline COMPORTAMIENTO & $\%$ \\
\hline Tapar recipientes de agua & $43 \%$ \\
\hline Usar larvicida & $42 \%$ \\
\hline Cambiar agua almacenada & $39 \%$ \\
\hline Vaciar agua estancada en el hogar & $38 \%$ \\
\hline Limpiar recipientes de agua & $36 \%$ \\
\hline Usar repelente & $28 \%$ \\
\hline Vaciar agua estancada en lugares comunes & $25 \%$ \\
\hline Usar condones en general & $21 \%$ \\
\hline Usar condones durante el embarazo & $14 \%$ \\
\hline
\end{tabular}

Al enumerar conductas preventivas según su nivel de efectividad para prevenir la reproducción de mosquitos y enfermedades como el Zika, casi 30 por ciento de los participantes consideró que fumigar su hogar y alrededores con insecticida estaba entre los tres comportamientos más efectivos, seguido por eliminar agua estancada (23 por ciento) y usar larvicida (22 por ciento) (ver Tabla 3). 
En comparación a los hombres, es significativamente más probable que las mujeres perciban limpiar recipientes de agua como uno de los tres comportamientos más efectivos (5 por ciento, $\mathrm{p}<0.05)$. Esta diferencia puede ser producto de los roles de género, ya que las mujeres, que suelen encargarse con mayor frecuencia de las labores domésticas, pueden estar más informadas de los beneficios de mantener limpios los recipientes de agua.

\section{TABLA 3. PORCENTAJE DE ENCUESTADOS QUE PERCIBEN EL SIGUIENTE COMPORTAMIENTO COMO UNA DE LAS TRES FORMAS MÁS EFECTIVAS PARA PREVENIR EL ZIKA}

\begin{tabular}{l|c}
\hline COMPORTAMIENTO & $\%$ \\
\hline Fumigar con insecticida (ej. Raid) & $29 \%$ \\
\hline Eliminar agua estancada & $23 \%$ \\
\hline Usar larvicida & $22 \%$ \\
\hline Despejar basura afuera de la casa & $19 \%$ \\
\hline Usar repelente & $19 \%$ \\
\hline Tapar contenedores o recipientes de agua & $16 \%$ \\
\hline Usar condones & $10 \%$ \\
\hline Usar rejillas en ventanas y puertas & $10 \%$ \\
\hline Usar mosquiteros en las camas & $9 \%$ \\
\hline Limpiar recipientes de agua & $8 \%$ \\
\hline Cambiar agua almacenada & $7 \%$ \\
\hline Usar espirales repelentes & $4 \%$ \\
\hline
\end{tabular}

\section{Hallazgos clave sobre comportamientos preventivos}

\section{Prácticas para prevenir picaduras de mosquitos y criaderos de mosquitos}

De las personas encuestadas:

- 55 por ciento declara haber hecho algo para prevenir picaduras de mosquito en los últimos treinta días, pero solo 24 por ciento declara haber llevado a cabo al menos una práctica preventiva de alta efectividad en ese tiempo.

- 42 por ciento dijo haber hecho algo para prevenir picaduras de mosquito en los últimos siete días, pero solo 24 por ciento declara haber llevado a cabo al menos una práctica preventiva de alta efectividad en ese tiempo.
Según las respuestas a una pregunta abierta, la Figura 2 muestra los comportamientos preventivos más frecuentes realizados en los últimos 30 días y últimos 7 días. El más reportado fue fumigar el hogar con insecticida en el hogar, mientras que el menos frecuente fue la limpieza comunitaria de agua estancada. Los comportamientos preventivos de mayor efectividad están marcados con un asterisco.

Más de un tercio (41 por ciento) de la muestra respondió no haber realizado ningún tipo de conducta preventiva en los últimos treinta días. El motivo más frecuente fue no sentir preocupación por las picaduras de mosquito (27 por ciento). Respecto al uso de repelente, la barrera más común para su uso fue su costo o dificultad de acceso, el cual fue mencionado por 16 por ciento de los encuestados que no habían usado repelente en los últimos siete días.

\section{FIGURA 2. COMPORTAMIENTOS PREVENTIVOS EN LOS ÚLTIMOS 30 Y 7 DÍAS}

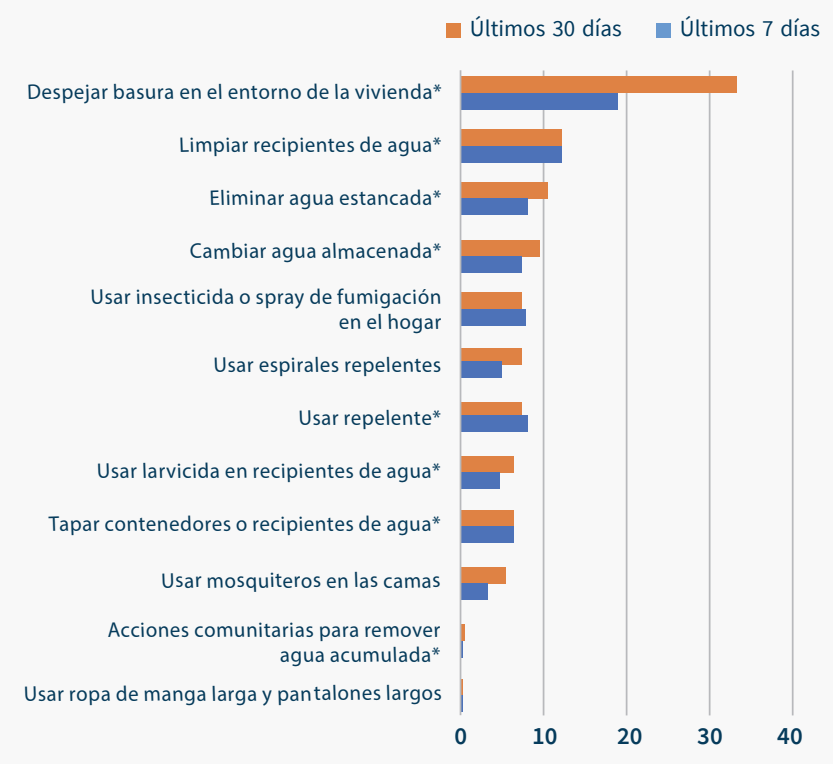

\section{Prácticas relacionadas con recipientes de agua}

Para entender la manera en que las personas limpian sus recipientes de agua, se les pidió a quienes lo habían hecho en los últimos siete días que especificaran cada paso que llevaron a cabo. La Tabla 4 muestra las acciones reportadas para limpiar recipientes de agua. Más de la mitad de quienes respondieron a esta pregunta (58 por ciento) reportaron haber agregado cloro al agua, lo que no corresponde a un método efectivo para eliminar larvas o pupas de mosquitos. Ningún encuestado respondió haber esperado algunos minutos para dejar actuar los productos de limpieza en la superficie del recipiente de agua. 
TABLA 4. PORCENTAJE DE ENCUESTADOS QUE LIMPIARON SU RECIPIENTE O CONTENEDOR DE AGUA CON LA SIGUIENTE ACCIÓN

\begin{tabular}{l|c} 
ACCIONES & $\%$ \\
\hline Aplicar cloro a las paredes del recipiente & $65 \%$ \\
\hline Agregarle cloro al agua & $58 \%$ \\
\hline Vaciar el recipiente & $40 \%$ \\
\hline Enjuagar el recipiente & $33 \%$ \\
\hline Cepillar el recipiente & $30 \%$ \\
\hline $\begin{array}{l}\text { Aplicar detergente a las paredes del recipiente } \\
\text { Dejar que los productos de limpieza hagan } \\
\text { efecto durante algunos minutos }\end{array}$ & $13 \%$ \\
\hline
\end{tabular}

Además de la encuesta, también se recogieron datos mediante la observación de recipientes de agua en las casas de los participantes. 462 de los 651 encuestados en la República Dominicana (71 por ciento) accedió a que un encuestador observe los recipientes o contenedores de agua de su hogar. Del total, 227 de los recipientes observados fueron piletas y 96 recipientes de agua de largo plazo (cotidianamente conocidos como tanques; ver Tabla 5). Los encuestadores observaron las tapas de los recipientes para evaluar su calidad. Para tapar un recipiente de manera efectiva, las tapas deben cubrir completamente el recipiente y encajar bien, evitando cualquier espacio abierto en toda la circunferencia; si el material de la tapa es plástico sólido, no debe estar doblado ni debe permitir que el agua se acumule en su superficie; si el material de la tapa es maleable, no debe tocar la superficie del agua para no arriesgar la formación de criaderos de mosquitos adicionales.

\begin{tabular}{l|c}
\multicolumn{2}{l}{ TABLA 5. OBSERVACIONES DE CONTENEDORES DE } \\
ALMACENAMIENTO \\
PILETAS & $\%$ \\
\hline Tapados & $77 \%$ \\
\hline Tapados de manera correcta & $60 \%$ \\
\hline \multicolumn{1}{|c}{ Se encontraron larvas de mosquitos } & $24 \%$ \\
\hline RECIPIENTES DE LARGO PLAZO & \\
\hline Tapados & $70 \%$ \\
\hline Tapados de manera correcta & $57 \%$ \\
\hline \multicolumn{2}{c}{ Se encontraron larvas de mosquitos } \\
\hline
\end{tabular}

No se encontraron diferencias significativas en la presencia de larvas entre piletas o recipientes que estaban tapados de manera correcta y aquellos que no estaban tapados o que estaban tapados de manera incorrecta, lo que indica un posible uso inconsistente de las tapas.

\section{Asociaciones entre determinantes de comportamiento y comportamientos preventivos $\$$}

Los resultados sugieren que el principal predictor para realizar comportamientos preventivos es tener conocimiento sobre comportamientos preventivos efectivos. Mediante el uso de una escala que mide el número de comportamientos preventivos conocidos, encontramos que un mayor conocimiento de comportamientos preventivos efectivos estaba relacionado significativamente con un mayor autorreporte de los siguientes comportamientos: tapar recipientes de agua, limpiar agua estancada, y uso de repelente. Según nuestros datos, es tres veces más probable que personas con un buen conocimiento del Zika -aquellos que conocen al menos seis comportamientos preventivos diferentes - lleven a cabo comportamientos preventivos que personas sin dicho conocimiento (48 por ciento vs. 16 por ciento). No obstante, cerca de la mitad de las personas con buen conocimiento de Zika en la República Dominicana no han llevado a cabo comportamientos preventivos. Por lo tanto, tener conocimiento del tema no basta para asegurar un comportamiento deseado.

Percibir los repelentes como un método efectivo para prevenir el Zika estaba relacionado significativamente con haber usado repelentes en los últimos 30 días. Las personas que clasificaron el uso de repelentes como uno de los tres métodos más efectivos tenían una probabilidad un 11 por ciento mayor de haberlos usado $(p<0.001)$.

Finalmente, las visitas a hogares por parte del equipo técnico o voluntarios de la comunidad para conversar sobre estrategias de prevención de mosquitos estaban relacionadas significativamente con un mayor conocimiento sobre el Zika y un mayor autorreporte de comportamientos preventivos. Las mujeres que recibieron a alguien en sus hogares para hablarles sobre métodos para prevenir mosquitos en el hogar y sus alrededores presentaron un incremento de seis puntos porcentuales den la probabilidad de saber que el Zika se puede transmitir sexualmente que aquellas mujeres que no recibieron visita, controlando por educación, edad, sexo, status socioeconómico, conocer a alguien que ha tenido Zika, y acceso a fuentes de información ( $p<0.05)$. Los hombres que recibieron a alguien en su hogar tenían un 13 por ciento mayor de probabilidad de saber que el Zika puede causar defectos congénitos ( $p .<0.05)$. Los participantes que recibieron a alguien en su hogar para hablar sobre métodos para prevenir mosquitos tenían un seis por ciento mayor de probabilidad de haber limpiado un recipiente de agua en los últimos siete días, y los hombres que recibieron a alguien tenían mayor probabilidad

$\S$ Los resultados de esta sección están basados en análisis de regresión logística. 
de cubrir los recipientes de agua en su hogar $(p<0.05)$. No se encontró una relación significativa entre visitas de hogar y uso de repelente.

\section{Conclusiones e implicancias para el futuro}

En las áreas estudiadas, la mayoría de la población conoce el virus del Zika. Sin embargo, a pesar de un alto nivel de conocimiento general, aún existe poca información sobre el modo en que el virus se transmite. Entre las personas que saben de que se transmite mediante mosquitos, pocas están informadas de que las picaduras pueden ocurrir a cualquier hora del día o la noche. Además, uno de cada cinco individuos que conoce el Zika no puede evaluar su nivel de riesgo frente al virus. Combinar mensajes sobre el Zika y el dengue puede elevar el nivel de conocimiento de la población sobre la transmisión del Zika mediante mosquitos, dado el alto nivel de conocimiento que existe sobre la transmisión del dengue mediante mosquitos. Además, pocas personas son conscientes de que el virus se puede transmitir mediante relaciones sexuales, y pocas personas conocen los efectos adversos sobre la salud que puede generar el Zika durante el embarazo. Si bien se estima que solo entre 4 y 5 por ciento de las transmisiones de Zika ocurren mediante relaciones sexuales, las implicancias de la transmisión del Zika durante el embarazo son muy graves-los programas deben continuar educando sobre la transmisión sexual del Zika como también de sus posibles consecuencias durante el embarazo. ${ }^{5,6}$

Mientras la mayoría de las personas conocen al menos una manera de prevenir el Zika de manera efectiva, existe un bajo nivel de conocimiento sobre la mayoría de los métodos efectivos de prevención, especialmente sobre métodos de protección personal. Las personas prácticamente no saben que usar condones y repelentes son mecanismos de prevención del Zika. En general, se percibe que los métodos de prevención efectivos son poco factibles, especialmente aquellos orientados a proteger a mujeres embarazadas y sus parejas, como el uso de repelentes y condones. ${ }^{7}$ Además, una gran parte de la población no intenta prevenir la reproducción de mosquitos ni sus picaduras. Entre las personas que sí realizan comportamientos preventivos, el comportamiento más frecuente - fumigar con insecticida en el hogar - tiene un efecto limitado para evitar la transmisión del Zika. ${ }^{8}$ Los programas deben priorizar actividades que aumenten el conocimiento de comportamientos específicos para prevenir el Zika -especialmente aquellos que han sido identificados como altamente efectivos contra enfermedades transmitidas por el mosquito Aedes aegypti. Se debe también destacar la efectividad de los diferentes comportamientos, y entregar instrucciones detalladas sobre aquellos que sean más complejos, como limpiar y tapar recipientes de agua. ${ }^{7,8}$ Para eliminar los criaderos de mosquitos de manera efectiva, es importante referirse detalladamente a pasos que suelen omitirse, como dejar que el cloro y los productos de limpieza hagan efecto en las paredes del recipiente por algunos minutos.

Los programas de prevención también deben intentar aumentar la factibilidad de los comportamientos preventivos efectivos, destacando su sencillez y eliminando barreras para llevarlos a cabo.

Finalmente, las visitas presenciales a los hogares siguen siendo un mecanismo favorable para estimular e informar a poblaciones en situaciones de alto riesgo y promover prácticas preventivas. 


\section{Referencias bibliográficas}

1 World Health Organization (WHO). 2016. Zika Strategic Response Plan. Geneva, Switzerland: WHO.

2 Haby, Michelle et al. 2018. "Prevalence of asymptomatic Zika virus infection: a systematic review," Bulletin of the World Health Organization 96: 402-413D

3 Centers for Disease Control and Prevention (CDC). 2018. Congenital Zika Syndrome \& Other Birth Defects. Available from www.cdc.gov/pregnancy/zika/testingfollow-up/zika-syndrome-birth-defects.html.

4 Health Communication Capacity Collaborative. 2016. Social and Behavior Change Communication for Emergency Preparedness Implementation Kit. Baltimore. Johns Hopkins University (JHU). Available from: http://healthcommcapacity.org/wp-content/ uploads/2017/01/SBCCforEP I-Kit.pdf.

5 Coelho, Falvio Codeco et al. 2016. "Higher incidence of Zika in adult women than adult men in Rio de Janeiro suggests a significant contribution of sexual transmission from men to women," International Journal of Infectious Diseases 51: 128-132.

6 Rao, Rashmi et al. 2017. "Zika risk and pregnancy in clinical practice: ongoing experience as the outbreak evolves," Obstetrics and Gynecology 129(6): 10981103.

7 Breakthrough ACTION+RESEARCH. 2018. Technical Specification Content Guide for Behaviors With a High Potential to Prevent Zika. Baltimore: JHU. Available from https://www.zikacommunicationnetwork.org/ resources/technical-specifications-content-guidebehaviors-high-potential-prevent-zika.

8 Breakthrough ACTION+RESEARCH. 2018. Zika Prevention Behavior Matrix. Baltimore: JHU. Available from https://www.zikacommunicationnetwork.org/ resources/zika-prevention-behavior-matrix.

\section{Agradecimientos}

Este informe de investigación describe el trabajo liderado por la Universidad de Tulane bajo el Proyecto Breakthrough RESEARCH, en colaboración con el Instituto de Medicina Tropical \& Salud Gobal de la Universidad Iberoamericana (UNIBE) de la República Dominicana. Este informe fue desarrollado por Martha Silva y Julia Fleckman de la Universidad de Tulane, junto a Kate Spielman del Population Council. Paul Hewett del Population Council y Arianna Serino de la Agencia para el Desarrollo Internacional de los Estados Unidos (The United States Agency for International Development - USAID) sirvieron de revisores técnicos. Heidi Worley de PRB apoyó el trabajo editorial y Katie Hoch realizó el diseño gráfico. Cristián Valenzuela tradujo el texto al español.

Cita sugerida:

Breakthrough RESEARCH. 2019. "Conocimiento, Actitudes y

Prácticas sobre el Zika: Implicancias Prográmaticas para Prevención del Zika en la República Domincana," Programmatic Research Brief. Washington, DC: Population Council.

Crédito fotográfico

BokehRD, Getty Images

() 2019 The Population Council. Todos los derechos reservados.

Breakthrough RESEARCH, Population Council

4301 Connecticut Ave., NW, Suite 280 | Washington, DC 20008 +1 2022379400 | breakthroughactionandresearch.org

BreakthroughRESEARCH@popcouncil.org

\section{USAID

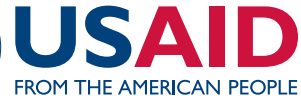

Breakthrough RESEARCH is made possible by the generous support of the American people through the United States Agency for International Development (USAID) under the terms of cooperative agreement no. AID-OAA-A-17-00018. The contents of this document are the sole responsibility of the Breakthrough RESEARCH and Population Council and do not necessarily reflect the views of USAID or the United States Government.
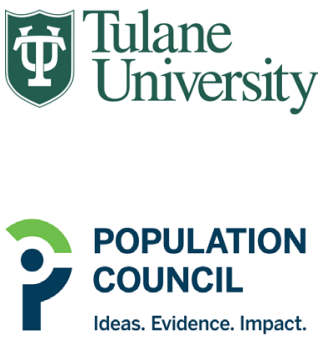

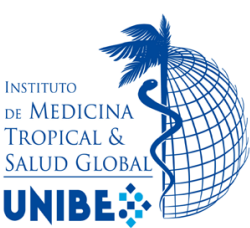

UNIBE:

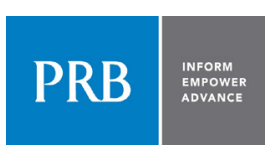

\section{Breakthrough RESEARCH

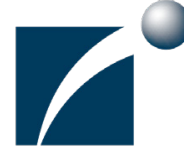

Breakthrough RESEARCH catalyzes social and behavior change (SBC) by conducting state-ofthe-art research and evaluation and promoting evidence-based solutions to improve health and development programs around the world. Breakthrough RESEARCH is a consortium led by the Population Council in partnership with Avenir Health, ideas42, Institute for Reproductive Health at Georgetown University, Population Reference Bureau, and Tulane University. 